\title{
Nonequilibrium critical dynamics of the three-dimensional gauge glass
}

\author{
Federico Romá and Daniel Domínguez \\ Centro Atómico Bariloche, R8402AGP San Carlos de Bariloche, Río Negro, Argentina
}

(Received 6 June 2008; revised manuscript received 4 September 2008; published 21 November 2008)

\begin{abstract}
We study the nonequilibrium aging behavior of the gauge glass model in three dimensions at the critical temperature. We perform Monte Carlo simulations with a Metropolis update, and correlation and response functions are calculated for different waiting times. We obtain a multiplicative aging scaling of the correlation and response functions, calculating the aging exponent $b$ and the nonequilibrium autocorrelation decay exponent $\lambda_{c} / z_{c}$. We also analyze the fluctuation-dissipation relationship at the critical temperature, obtaining the critical fluctuation-dissipation ratio $X_{\infty}$. By comparing our results with the aging scaling reported previously for a model of interacting flux lines in the vortex-glass regime, we found that the exponents for both models are very different.
\end{abstract}

DOI: 10.1103/PhysRevB.78.184431

PACS number(s): 75.50.Lk, 74.25.Qt, 74.40.+k, 05.70.Ln

\section{INTRODUCTION}

The possibility of a vortex-glass phase in high- $T_{c}$ superconductors was proposed in 1989 by Fisher and co-workers. ${ }^{1-3}$ Throughout the years, two possible scenarios have been discussed: either there is a continuous phase transition from the vortex liquid to the vortex glass at a finite critical temperature ${ }^{1,4-7}$ or there is a crossover temperature $^{8-10}$ below which the vortex liquid freezes in a glassy regime. While early theoretical ${ }^{4,5}$ and experimental studies $^{6}$ supported the existence of a finite temperature critical point, more recent theoretical arguments tend to favor the freezing scenario for the vortex liquid at a crossover temperature. ${ }^{89}$ Experimentally, it is difficult to differentiate between a critical point and a strong crossover in near equilibrium measurements in superconductors with random pinning. ${ }^{7,10}$ The aim of this work is to show that the study of the nonequilibrium dynamics can help us to clearly distinguish among these two scenarios. To this end, we will study nonequilibrium correlation and response functions in a model for the vortex-glass transition that has a finite temperature critical point (the unscreened gauge glass model) ${ }^{4}$ and compare with previous results in a model that has a crossover freezing transition to a glassy regime [the model of interacting flux lines (IFL)]. ${ }^{9}$

The gauge glass $(\mathrm{GG})$ model $^{4,5,11}$ is a paradigmatic model for the vortex-glass phase transition. ${ }^{2,3}$ It consists of the $X Y$ model with a random gauge potential vector. The GG is assumed to represent the physics of a superconductor with disorder at relatively high magnetic fields. ${ }^{2-4}$ It has been found that this model has a finite $T_{c}$ in three dimensions ${ }^{5,12,13}$ and several numerical works have studied its critical behavior, considering both equilibrium properties as well as dynamical and transport properties. ${ }^{4,5,11-18}$ Early experiments ${ }^{6}$ on the scaling of current-voltage curves in high- $T_{c}$ superconductors obtained critical exponents $\nu$ and $z$ in reasonable agreement with the exponents obtained in simulations in the GG. However, in 1995 Bokil and Young ${ }^{8}$ showed that $T_{c}=0$ in three dimensions when magnetic screening in a finite length scale $\lambda$ is included in the GG (by adding fluctuations of the vector potential)..$^{8,19-21}$ Later experiments also showed that the current-voltage curves do not scale as expected, ${ }^{10}$ while other experimental groups report good scaling with the GG exponents. ${ }^{7}$ Since all superconductors have a finite screening length $\lambda$ (the London penetration depth), the findings in Ref. 8 discard the scenario of a finite temperature critical point. Taking into account this result, Zimányi and co-workers ${ }^{9,22}$ considered a model of IFL with finite interaction length $\lambda$ and in the presence of quenched disorder. ${ }^{23}$ They found in this model that upon decreasing temperature the vortexglass-like criticality is arrested at a crossover temperature signaling a freezing of flux lines. Below this temperature the time scales grow very quickly with a dynamical behavior similar to what is found in structural ("window") glasses. ${ }^{9,24}$

This later result brings into attention the importance of the nonequilibrium behavior of vortex glasses as well as its possible relationship with the dynamical behavior of other glassy systems. In the recent years, there has been some progress in the general understanding of glasses through the study of their out-of-equilibrium dynamics. ${ }^{25} \mathrm{~A}$ characteristic of relaxing glassy systems is the loss of stationarity reflected by their aging properties, meaning that the dynamics of the system depends on the time elapsed after the preparation of the sample, $t_{w} \cdot{ }^{25}$ As a consequence, dynamic correlation functions depended on two times, the "waiting time" $t_{w}$ and the time $t$ elapsed during the measurement. Also the linearresponse functions show aging effects, being dependent on $t_{w}$ and $t$, and they are anomalous in the sense that they are not related to their associated correlation functions by the equilibrium fluctuation-dissipation theorem (FDT). ${ }^{25,26}$ The standard protocol for the study of aging in glassy systems $\mathrm{s}^{25,26}$ consists in preparing the sample at a high temperature $\left(T_{\text {start }} \rightarrow \infty\right)$ and then to quench it to a final low temperature $T_{f}$. At $T_{f}$, two-time-correlation functions $C\left(t, t_{w}\right)$ and response functions $R\left(t, t_{w}\right)$ are analyzed, where the measurement starts for $t \geq t_{w}$. In several systems a simple aging law with $C\left(t, t_{w}\right) \sim C_{\mathrm{ag}}\left(t / t_{w}\right)$ is typically found. ${ }^{26}$ On the other hand, a "multiplicative" aging law of the form $C\left(t, t_{w}\right)$ $\sim t_{w}^{-\alpha} C_{\mathrm{ag}}\left(t / t_{w}\right)$ has been found in polymers in random media ${ }^{27}$ and in systems that are quenched at the critical temperature, such as ferromagnetic spin models ${ }^{28-33}$ and the Ising spin glass. ${ }^{34,35}$

In the case of vortices in superconductors with quenched disorder, Bustingorry et al. ${ }^{24}$ studied the nonequilibrium ag- 
ing dynamics of the IFL model. They found that it can be characterized by "multiplicative aging," with a scaling form similar to what is found in polymers in random media. ${ }^{27}$ In order to compare with this result, we will study here the aging and nonequilibrium dynamics of the GG model at the critical temperature, calculating two-time-correlation and response functions. Being at the critical point, a multiplicative aging law is expected, which in form should be similar to the type of aging observed numerically in the IFL. ${ }^{24}$ Our aim is to compare the aging exponents of the GG at $T_{c}$ with the ones obtained for the IFL in Ref. 24. As we will show here, they turn out to be very different, a result that indicates that nonequilibrium aging experimental measurements could clearly distinguish among the two scenarios discussed in the first paragraph.

The paper is organized as follows. In Sec. II we present the model Hamiltonian and the simulation method. In Sec. III we define the observables to be calculated. In Sec. IV we present our results for the correlation and response functions after a critical quench in the GG and we analyze their scaling with $t_{w}$. Finally in Sec. V we discuss our results comparing with the disorder-free three-dimensional (3D) $X Y$ model, the 3D Ising spin glass (ISG), and the IFL.

\section{MODEL AND MONTE CARLO SIMULATIONS}

The Hamiltonian of the 3D GG model ${ }^{4}$ is given by

$$
H=-J \sum_{(i, j)} \cos \left(\theta_{i}-\theta_{j}-A_{i, j}\right),
$$

where $\theta_{i}$ represents the superconducting phase at site $i$, and we sum over nearest neighbors $(i, j)$ on a cubic lattice of linear size $L\left(N=L^{3}\right)$. The $A_{i, j}$ are quenched random variables uniformly distributed in the $[0,2 \pi]$ interval $\left(A_{i, j}=-A_{j, i}\right)$ and $J$ is the coupling between nearest neighbors. The phases $\theta_{i}$ can be represented as the angle of classical two-dimensional spins of unit length, $S_{i}=\left(\cos \theta_{i}\right.$, $\left.\sin \theta_{i}\right)$. In this work periodic boundary conditions are applied in the phases $\theta_{i}$. The energy is normalized in units of $J$, temperature in units of $J / k_{B}$, and time scales are measured in number of Monte Carlo steps (for full sweep for the $N$ sites).

The out-of-equilibrium protocol used in this work consists of a quench at time $t=0$ from a $T=\infty$ state to the critical temperature $T_{c}$. For the $3 \mathrm{D}$ GG model, the critical temperature is $T_{c}=0.46(1)$, according to Ref. 17. From this initial condition different two-time quantities are analyzed, which depend on both the waiting time $t_{w}$, when the measurement begins, and a given time $t>t_{w}$.

For the Monte Carlo simulation local changes in the phases $\theta_{i} \rightarrow \theta_{i}^{\prime}$ are accepted with probability given by the Metropolis rate,

$$
p\left(\theta_{i} \rightarrow \theta_{i}^{\prime}\right)=\min \{1, \exp (-\beta \Delta H)\} .
$$

Here $\beta$ is the inverse temperature and $\Delta H$ is the energy difference corresponding to the proposed phase change. In equilibrium simulations, the acceptance window for $\theta_{i}^{\prime}$ is usually chosen less than $2 \pi$ and dependent on temperature in order to optimize the updating procedure. Because we are interested in studying a nonequilibrium process, for local phase changes we will use the full $2 \pi$ acceptance angle window for the new phases $\theta_{i}^{\prime}$, following the criterion used in Refs. 17 and 18. This is done in order to avoid the possibility that a limited acceptance angle might introduce an artificial temperature dependence in the relaxation.

\section{OBSERVABLES}

For simplicity, we will focus on the study of the two-timeautocorrelation function defined as

$$
C\left(t, t_{w}\right)=\frac{1}{N}\left[\left\langle\sum_{i=1}^{N} \cos \left[\theta_{i}(t)-\theta_{i}\left(t_{w}\right)\right]\right\rangle\right]_{\mathrm{av}},
$$

where $\langle\cdots\rangle$ indicates an average over different thermal histories (different initial configurations and realizations of the thermal noise) and $[\cdots]_{\mathrm{av}}$ represents a disorder average over different samples (different realizations of $A_{i, j}$ ). The corresponding two-time linear autoresponse function is

$$
R\left(t, t_{w}\right)=\frac{1}{N}\left[\left\langle\sum_{i=1}^{N} \frac{\delta \boldsymbol{S}_{i}(t)}{\delta \boldsymbol{h}_{i}\left(t_{w}\right)}\right\rangle\right]_{\mathrm{av}} .
$$

In this case, the system is perturbed by applying an infinitesimal external field $\boldsymbol{h}_{i}$ conjugated to $\boldsymbol{S}_{i}$. This corresponds to a perturbing term in the Hamiltonian $\Delta H=-\sum_{i} \boldsymbol{h}_{i} \cdot \boldsymbol{S}_{i}$. In numerical simulations it is more convenient to calculate the integrated responses: either the thermoremanent response $M_{\mathrm{TRM}}\left(t, t_{w}\right)$ or the zero-field-cooled response $M_{\mathrm{ZFC}}\left(t, t_{w}\right)$, which are obtained by switching on the perturbation only for times $t<t_{w}$ and $t>t_{w}$, respectively. If we define the reduced integrated responses by

$$
\begin{aligned}
\rho_{\mathrm{TRM}}\left(t, t_{w}\right) & =\frac{T}{h} M_{\mathrm{TRM}}\left(t, t_{w}\right), \\
\rho_{\mathrm{ZFC}}\left(t, t_{w}\right) & =\frac{T}{h} M_{\mathrm{ZFC}}\left(t, t_{w}\right),
\end{aligned}
$$

then we can relate these functions to $R\left(t, t_{w}\right),{ }^{29}$

$$
\begin{gathered}
\rho_{\mathrm{TRM}}\left(t, t_{w}\right)=T \int_{0}^{t_{w}} d u R(t, u), \\
\rho_{\mathrm{ZFC}}\left(t, t_{w}\right)=T \int_{t_{w}}^{t} d u R(t, u) .
\end{gathered}
$$

If the perturbing field in each site, $\boldsymbol{h}_{i}$, is random and its two components are independently drawn from a bimodal distribution $\pm h$, both reduced integrated responses are given by ${ }^{31}$

$$
\rho\left(t, t_{w}\right)=\frac{T}{h^{2} N}\left[\left\langle\sum_{i=1}^{N} \boldsymbol{h}_{i} \cdot \boldsymbol{S}_{i}\right\rangle\right]_{\mathrm{av}} .
$$

In this work we show simulation results for systems of size $L=30$ at the temperature $T_{c}=0.46$. We take the disorder average over 60 samples and, for each sample, we carry out a number of ten thermal histories. The magnitudes of the external fields used for the calculation of the response function were $h=0.1$ and $h=0.05$. 


\section{CRITICAL QUENCH}

At the critical temperature $T_{c}$ the equilibrium autocorrelation relaxation time $\tau_{c}$ increases with the size as $\tau_{c} \sim L^{z_{c}}$, where $z_{c}$ is the dynamical critical exponent. For simple ferromagnetic systems, it is known that in a critical quench spatial correlations over a length scale of $l \sim t^{1 / z_{c}}$ are just as in the equilibrium critical state. This means that the system appears critical on scales smaller than $l$, while it appears disordered on larger scales. ${ }^{29}$

Considering this, autocorrelation function (3) is expected to behave as ${ }^{29,35}$

$$
C\left(t, t_{w}\right)=t_{w}^{-b} f_{C}\left(\frac{t}{t_{w}}\right) .
$$

For $\tau \ll t_{w}\left(\tau=t-t_{w}\right)$, the critical scaling function $f_{C}(x)$ behaves as

$$
f_{C}(x) \sim[x-1]^{-b} .
$$

For $d$ dimensional spin glasses it has been found ${ }^{34,36}$ that

$$
b=\frac{d-2+\eta}{2 z_{c}} .
$$

Here $\eta$ is the static critical exponent associated to the paircorrelation function. Then, for $\tau \ll t_{w}$ the autocorrelation function is

$$
C\left(t, t_{w}\right) \sim \tau^{-b} .
$$

On the other hand, when $\tau \gg t_{w}$ we have

$$
f_{C}(x) \sim A_{C} x^{-\lambda} C^{\prime z},
$$

where $A_{C}$ is a constant and $\lambda_{C}$ is known as the autocorrelation exponent. ${ }^{34}$

Figure 1(a) shows the behavior of the autocorrelation function $C\left(t, t_{w}\right)$ vs $\tau$ for eight different waiting times $t_{w}$. For $\tau \ll t_{w}$, we can fit a power-law behavior, $\tau^{-b}$, with exponent $b \approx 0.06$, as shown in the plot. For $\tau>t_{w}$ we observe that the correlation function shows aging since it depends strongly on the waiting time $t_{w}$. In the inset of Fig. 1(b) we show the result of assuming "simple aging" by plotting $C$ as a function of the ratio $t / t_{w}$. We observe that the data do not show a good collapse in a single curve in this case. A good data collapse can be obtained if the multiplicative aging scaling form of Eq. (10) is assumed. In Fig. 1(b), we show the plot of $t_{w}^{b} C$ vs $t / t_{w}$, obtaining good scaling with $b=0.06(1)$. This value is in good agreement with Eq. (12): if we take from previous simulations of the 3D GG model the values $\eta=-0.47(2)$ (Ref. 17) and $z_{c}=4.5(1)$ (Ref. 18) we obtain $b=0.059(4)$. We now consider the case of $\tau \gg t_{w}$. In Fig. 1(b) we show that in this case the asymptotic behavior of $C$ has a power-law form consistent with Eq. (14). We obtain $A_{C}=1.55(5)$ and $\lambda_{C} / z_{c}$ $=0.49(2)$.

We now consider the autoresponse function. In this case the scaling equation is ${ }^{29}$

$$
R\left(t, t_{w}\right)=t_{w}^{-1-a} f_{R}\left(\frac{t}{t_{w}}\right) .
$$

For $\tau \gg t_{w}$ is expected that
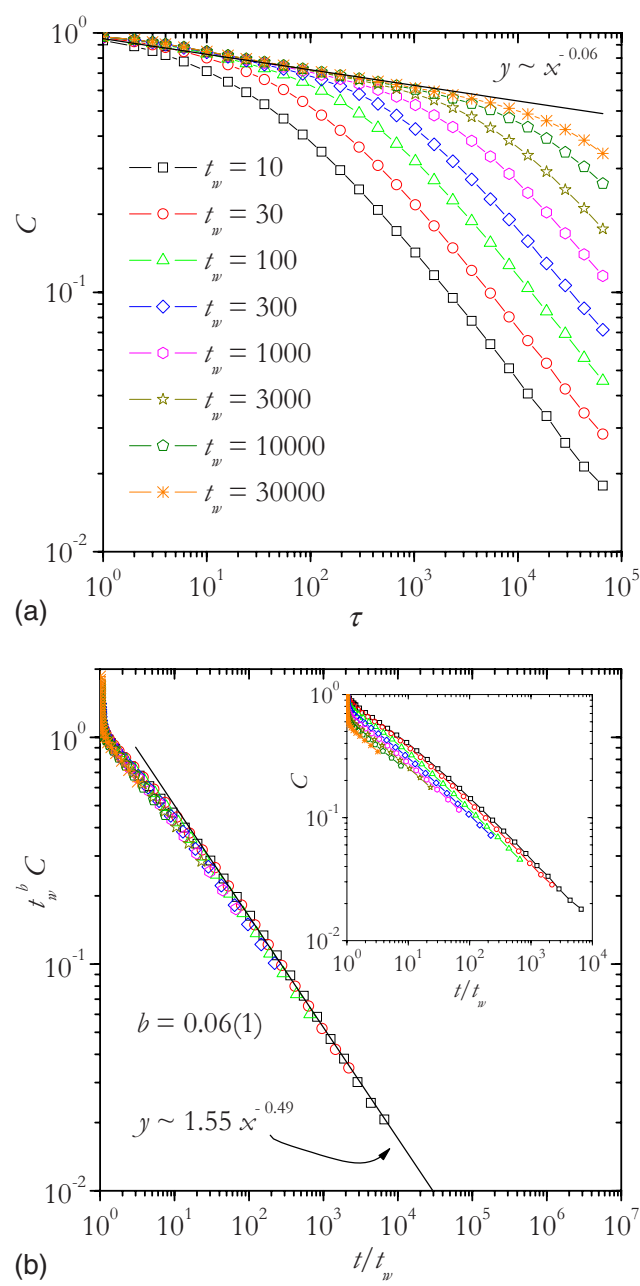

FIG. 1. (Color online) (a) Autocorrelation function $C$ vs $\tau$ for eight different waiting times as indicated. (b) Data collapsing. Inset: autocorrelation function $C$ vs $t / t_{w}$. Time scales are measured in number of Monte Carlo steps (for full sweep for the $N$ sites).

$$
f_{R}(x) \sim A_{R} x^{-\lambda_{R} / z_{c}},
$$

where $A_{R}$ is a constant amplitude. Then, if we consider Eq. (7), we obtain the thermoremanent reduced integrated response,

$$
\rho_{\mathrm{TRM}}\left(t, t_{w}\right)=t_{w}^{-a} f_{\rho}\left(\frac{t}{t_{w}}\right),
$$

where

$$
f_{\rho}(x) \sim A_{\rho} x^{-\lambda_{R} / z_{c}}
$$

and $A_{\rho}$ is a constant. For critical systems it is expected that $a=b$ and $\lambda_{C}=\lambda_{R}$.

In Fig. 2(a) we show the behavior of $\rho_{\mathrm{TRM}}$ function vs $\tau$ for different waiting times and $h=0.05$, observing that the response function also shows aging. In the inset of Fig. 2(b) we show $\rho_{\mathrm{TRM}}$ vs $t / t_{w}$ : no data collapse is observed. In Fig. 2(b) we plot $t_{w}^{a} \rho_{\mathrm{TRM}}$ vs $t / t_{w}$, obtaining good data collapse for $a=0.06(1)$. The asymptotic behavior observed for large temporal separations $\left(\tau \gg t_{w}\right)$ can be fitted with a power law with $A_{\rho}=0.18(2)$ and $\lambda_{R} / z_{c}=0.52(2)$. 

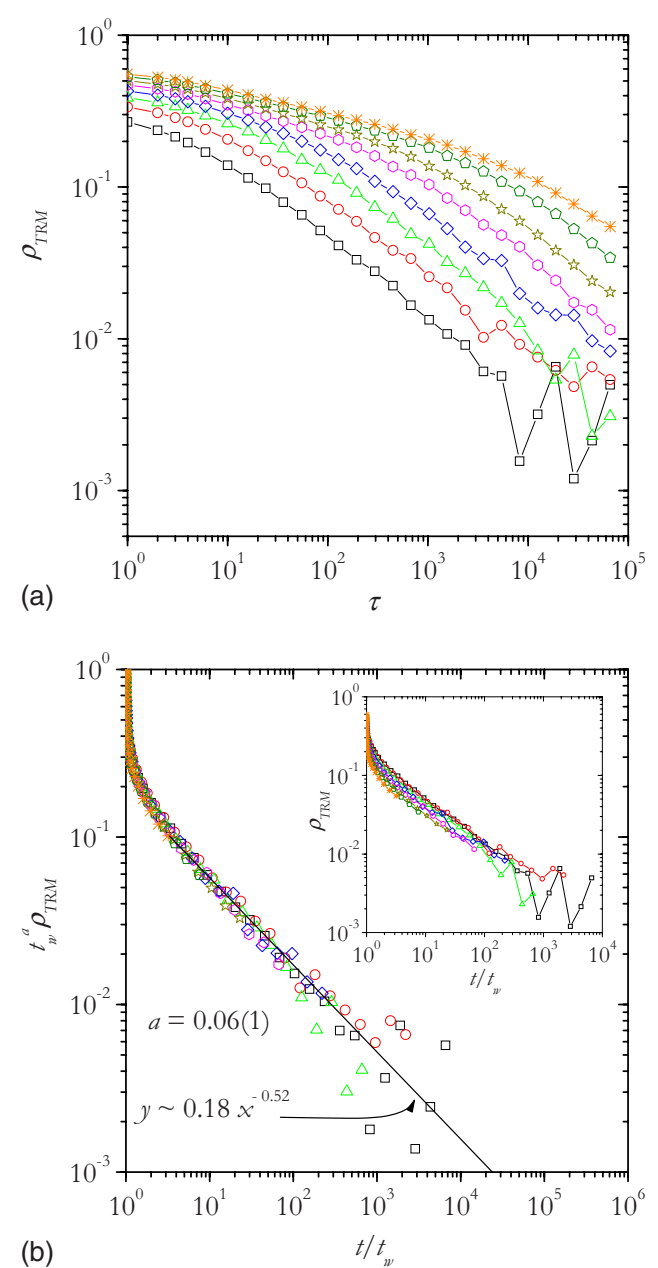

FIG. 2. (Color online) (a) Function $\rho_{\text {TRM }}$ vs $\tau$ for different waiting times and $h=0.05$. All symbols are the same as in Fig. 1(a). (b) Data collapsing. Inset: function $\rho_{\mathrm{TRM}} \mathrm{vs} t / t_{w}$ for different waiting times and $h=0.05$.

At thermodynamic equilibrium the FDT is satisfied, and therefore it is possible to draw a simple expression to relate the correlation with the thermoremanent reduced integrated response,

$$
\rho_{\mathrm{TRM}}\left(t-t_{w}\right)=C\left(t-t_{w}\right) .
$$

Then, for a parametric plot of $\rho_{\text {TRM }}$ vs $C$, we should obtain a straight line of slope +1 in equilibrium. On the other hand, for a nonequilibrium process the FDT is not fulfilled, and it has been proposed a generalized relation of the form

$$
\rho_{\mathrm{TRM}}\left(t, t_{w}\right)=X\left(t, t_{w}\right) C\left(t, t_{w}\right),
$$

where $X\left(t, t_{w}\right)$ is called the fluctuation-dissipation ratio (FDR). ${ }^{37}$ For $1 \ll t_{w} \ll t$ we can estimate the limit

$$
X_{\infty}=\lim _{t_{w} \rightarrow \infty} \lim _{t \rightarrow \infty} X\left(t, t_{w}\right) .
$$

For a critical quench, $X_{\infty}$ is expected to be universal in the sense that it does not depend either on the initial conditions or on the details of the dynamics. ${ }^{30}$

Figure 3 shows the $t$-parametric plot of $\rho_{\mathrm{TRM}}\left(t, t_{w}\right)$ vs $C\left(t, t_{w}\right)$ for different waiting times $t_{w}$. In the quasiequilibrium

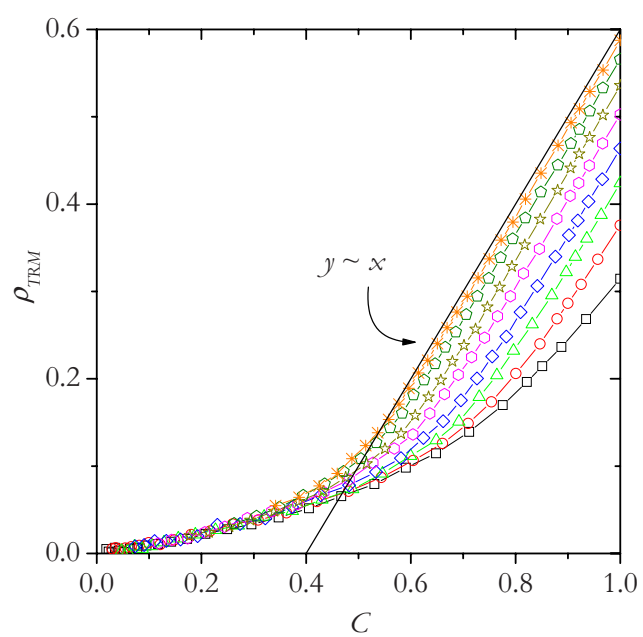

FIG. 3. (Color online) FDT plot for different waiting times and $h=0.05$. All symbols are the same as in Fig. 1(a).

regime $\left(\tau \ll t_{w}\right)$ the FDT holds and $X=1$. This is observed in Fig. 3 where for large $t_{w}$ the slope in the FDT plot tends to 1 . On the other hand, when $1 \ll t_{w} \ll t$, we observe that $X<1$ and FDT is violated. From Eqs. (10), (14), (16), (17), and (19), it is possible to demonstrate that

$$
X_{\infty}=A_{\rho} / A_{C} .
$$

From the amplitudes $A_{C}$ and $A_{\rho}$ calculated previously we obtain $X_{\infty}=0.12(2)$.

We can obtain $X_{\infty}$ numerically from the FDT plot of Fig. 3 in two different ways. First, in Fig. 4(a) we show a data collapse plotting $\rho_{\mathrm{TRM}}\left(t, t_{w}\right) t_{w}^{a}$ vs $C\left(t, t_{w}\right) t_{w}^{b}$. The slope near the origin corresponds to the limit of FDR, obtaining $X_{\infty}$ $=0.12(1)$. Second, in Fig. 4(b) we plot the ratio $t_{w}^{a} \rho_{\text {TRM }} / t_{w}^{b} C$ vs $t_{w}^{b} C$ for all times $t$ and $t_{w}$. We see that the ordinate at the origin is compatible with $X_{\infty} \approx 0.12$.

\section{DISCUSSION}

We have therefore obtained the exponents that characterize the aging scaling of the 3D GG model at the critical temperature, obtaining $b=0.06(1), \quad \lambda_{C} / z_{c}=0.49(2), \quad a$ $=0.06(1), \lambda_{R} / z_{c}=0.52(2)$, and $X_{\infty}=0.12(1)$. A comparison with results in the corresponding model without disorder, i.e., the 3D $X Y$ model, shows that the exponents are very different, as expected. Abriet and Karevski ${ }^{33}$ found $a=b$ $=1 / 2$ and $\lambda_{C} / z=\lambda_{R} / z=1.34$, which show that in the disorder-free case the multiplicative scaling of aging is stronger (i.e., much larger values of $a$ and $b$ ). Also a very different limit of the FDR, $X_{\infty}=0.43(4)$, was obtained, as expected since they should belong to different universality classes.

On the other hand, the critical aging of the GG turns out to be very similar to results found in the $3 \mathrm{D}$ ISG. ${ }^{34,35}$ It has been found for a binary distribution in the exchange couplings, $\quad a=0.060(4), \quad b=0.056(3), \quad \lambda_{C} / z=0.362(5), \quad \lambda_{R} / z$ $=0.38(2)$, and $X_{\infty}=0.12(1)$, and for a Gaussian distribution in the exchange couplings, $a=0.044(1), b=0.043(1), \lambda_{C} / z$ $=0.320(5), \lambda_{R} / z=0.33(2)$, and $X_{\infty}=0.09(1) .{ }^{34}$ As we can see, the aging exponents are slightly smaller in the 3D ISG 


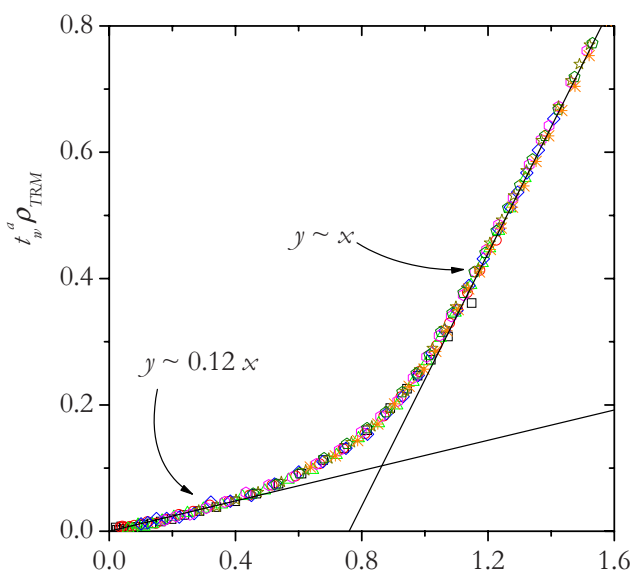

(a)

$t_{w}^{b} C$

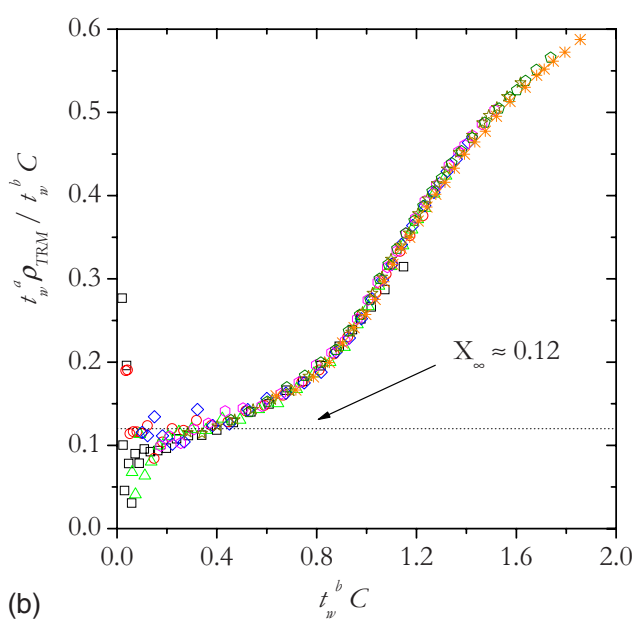

FIG. 4. (Color online) (a) Data collapsing for all curves in Fig. 3. (b) Plot of ratio $t_{w}^{a} \rho_{\mathrm{TRM}} / t_{w}^{b} C$ vs $t_{w}^{b} C$. All symbols are the same as in Fig. 1(a).

(meaning a weaker multiplicative aging), but the values of $X_{\infty}$ are comparable within the statistical error with the GG. It has been argued in Refs. 28-30 that $X_{\infty}$ is a novel universal quantity of nonequilibrium critical dynamics. It is interesting to observe that spin-glass models such as the ISG and GG have similar values of $X_{\infty}$. However, let us remind that most work on the equilibrium properties of the GG and the ISG shows evidence that they are in different universality classes. $5,11,12,15,18$

As discussed in Sec. I, two types of behaviors are predicted for the vortex-glass transition: either a finite temperature critical point as described by the GG or a crossover temperature as found in the IFL. The work of Bokil and
Young $^{8}$ discards the first scenario. However, from the experimental point of view, the issue is not clearly resolved since several experiments show reasonably good scaling at a transition temperature. ${ }^{7}$ Comparing our results with the behavior found by Bustingorry et al. ${ }^{24}$ in the IFL, we believe that a study of the nonequilibrium dynamics of the vortex-glass transition will be able to clearly distinguish between the two models. In simulations of the IFL, off-equilibrium correlation and response functions are found to scale as $B\left(t, t_{w}\right)$ $\sim t_{w}^{\alpha} \tilde{B}\left(t / t_{w}\right)$, and also it is found that $\widetilde{B}(x) \sim x^{\alpha}$ for $x \gg 1 .^{24}$ The first thing to notice when comparing with critical aging in the GG is that, in the IFL, the corresponding multiplicative aging exponent $b$ and the autocorrelation exponent $\lambda_{C} / z$ coincide $b=\lambda_{C} / z=\alpha$ (and similarly for the response exponents, $\left.a=\lambda_{R} / z=\alpha\right)$. The second main difference is that the multiplicative aging in the IFL is very strong, $b_{\mathrm{IFL}}=\alpha$ $\approx 0.25-0.35$, when compared with the GG at the critical point, $b_{\mathrm{GG}} \approx 0.06$. In the IFL the competition between the elasticity of each flux line and the randomness of the pinning potential leads to a very slow dynamics and strong aging with a large $\alpha$ exponent. Moreover, $\alpha$ depends with the disorder strength in the IFL, being larger for stronger disorder.

In this work we have analyzed the correlation and integrated response functions defined in Eqs. (3) and (9) since they are easy to compute numerically. In experiments in superconductors, correlation (response) functions of other observables such as magnetization (magnetic susceptibility) and voltage (resistance) are, of course, more available. Aging scaling similar to Eqs. (10) and (16) will also be expected in these other physical quantities. For example, one possible way of observing experimentally the out-of-equilibrium dynamics of the vortex glass is monitoring the magnetic relaxation as done in Ref. 38 for granular samples. Another possibility is to perform electrical transport experiments near the transition temperature following a protocol similar to the one used by Vaknin et al. ${ }^{39}$ to study the electron glass regime in Anderson insulators. In either case, an analysis of the multiplicative aging exponent will clearly distinguish between the two different models $\left(b_{\mathrm{IFL}} \approx 0.25-0.35\right.$ or $\left.b_{\mathrm{GG}} \approx 0.06\right)$.

\section{ACKNOWLEDGMENTS}

We acknowledge support from CNEA (Argentina) and from CONICET (Argentina) under Project No. PIP5596. F.R. thanks Universidad Nacional de San Luis (Argentina) under Project No. 322000 and acknowledges support from CONICET under Project No. PIP6294. D.D. acknowledges support from ANPCyT (Argentina) under Projects No. PICT2003-13829 and No. PICT2003-13511.
${ }^{1}$ M. P. A. Fisher, Phys. Rev. Lett. 62, 1415 (1989); D. S. Fisher, M. P. A. Fisher, and D. A. Huse, Phys. Rev. B 43, 130 (1991).

${ }^{2}$ G. Blatter, M. V. Feigel'man, V. B. Geshkenbein, A. I. Larkin, and V. M. Vinokur, Rev. Mod. Phys. 66, 1125 (1994).

${ }^{3}$ T. Nattermann and S. Scheidl, Adv. Phys. 49, 607 (2000).
${ }^{4}$ D. A. Huse and H. S. Seung, Phys. Rev. B 42, 1059 (1990).

${ }^{5}$ J. D. Reger, T. A. Tokuyasu, A. P. Young, and M. P. A. Fisher, Phys. Rev. B 44, 7147 (1991).

${ }^{6}$ R. H. Koch, V. Foglietti, W. J. Gallagher, G. Koren, A. Gupta, and M. P. A. Fisher, Phys. Rev. Lett. 63, 1511 (1989). 
${ }^{7}$ A. M. Petrean, L. M. Paulius, W.-K. Kwok, J. A. Fendrich, and G. W. Crabtree, Phys. Rev. Lett. 84, 5852 (2000).

${ }^{8}$ H. S. Bokil and A. P. Young, Phys. Rev. Lett. 74, 3021 (1995).

${ }^{9}$ C. Reichhardt, A. van Otterlo, and G. T. Zimányi, Phys. Rev. Lett. 84, 1994 (2000).

${ }^{10}$ D. R. Strachan, M. C. Sullivan, P. Fournier, S. P. Pai, T. Venkatesan, and C. J. Lobb, Phys. Rev. Lett. 87, 067007 (2001); I. L. Landau and H. R. Ott, Phys. Rev. B 65, 064511 (2002).

${ }^{11}$ A. Houghton and M. A. Moore, Phys. Rev. B 38, 5045 (1988); M. A. Moore and S. Murphy, ibid. 42, 2587 (1990).

${ }^{12}$ M. J. P. Gingras, Phys. Rev. B 44, 7139 (1991); 45, 7547 (1992).

${ }^{13}$ M. Cieplak, J. R. Banavar, and A. Khurana, J. Phys. A 24, L145 (1991).

${ }^{14}$ J. Maucourt and D. R. Grempel, Phys. Rev. B 58, 2654 (1998).

${ }^{15}$ T. Olson and A. P. Young, Phys. Rev. B 61, 12467 (2000).

${ }^{16}$ H. G. Katzgraber and A. P. Young, Phys. Rev. B 64, 104426 (2001); 66, 224507 (2002).

${ }^{17}$ H. G. Katzgraber and I. A. Campbell, Phys. Rev. B 69, 094413 (2004).

${ }^{18}$ H. G. Katzgraber and I. A. Campbell, Phys. Rev. B 72, 014462 (2005).

${ }^{19}$ C. Wengel and A. P. Young, Phys. Rev. B 54, R6869 (1996).

${ }^{20}$ C. Wengel and A. P. Young, Phys. Rev. B 56, 5918 (1997).

${ }^{21}$ J. Kisker and H. Rieger, Phys. Rev. B 58, R8873 (1998).

${ }^{22}$ A. van Otterlo, R. T. Scalettar, and G. T. Zimányi, Phys. Rev. Lett. 81, 1497 (1998).

${ }^{23}$ It is worth mentioning that in simulations of a model of flux lines with long-range interactions $(\lambda=\infty)$ a finite critical temperature was found, but with critical exponents different from the GG.
See A. Vestergren, J. Lidmar, and M. Wallin, Phys. Rev. Lett. 88, 117004 (2002).

${ }^{24}$ S. Bustingorry, L. F. Cugliandolo, and D. Domínguez, Phys. Rev. Lett. 96, 027001 (2006); Phys. Rev. B 75, 024506 (2007).

${ }^{25}$ L. F. Cugliandolo, in Slow Relaxations and Nonequilibrium Dynamics in Condensed Matter, edited by J.-L. Barrat, J. Dalibard, M. Feigel'man, and J. Kurchan (Springer, Berlin, 2002).

${ }^{26}$ A. Crisanti and F. Ritort, J. Phys. A 36, R181 (2003).

${ }^{27}$ H. Yoshino, Phys. Rev. Lett. 81, 1493 (1998).

${ }^{28}$ P. Calabrese and A. Gambassi, J. Phys. A 38, R133 (2005).

${ }^{29}$ C. Godrèche and J. M. Luck, J. Phys.: Condens. Matter 14, 1589 (2002).

${ }^{30}$ C. Godrèche and J. M. Luck, J. Phys. A 33, 1151 (2000); 33, 9141 (2000).

${ }^{31}$ L. Berthier, P. C. W. Holdsworth, and M. Sellito, J. Phys. A 34, 1805 (2001).

${ }^{32}$ S. Abriet and D. Karevski, Eur. Phys. J. B 37, 47 (2004).

${ }^{33}$ S. Abriet and D. Karevski, Eur. Phys. J. B 41, 79 (2004).

${ }^{34}$ M. Henkel and M. Pleimling, Europhys. Lett. 69, 524 (2005).

${ }^{35}$ M. Pleimling and I. A. Campbell, Phys. Rev. B 72, 184429 (2005).

${ }^{36}$ A. T. Ogielski, Phys. Rev. B 32, 7384 (1985).

${ }^{37}$ L. F. Cugliandolo and J. Kurchan, J. Phys. A 27, 5749 (1994).

${ }^{38}$ E. L. Papadopoulou, P. Nordblad, P. Svedlindh, R. Schöneberger, and R. Gross, Phys. Rev. Lett. 82, 173 (1999); E. L. Papadopoulou, P. Svedlindh, and P. Nordblad, Phys. Rev. B 65, 144524 (2002).

${ }^{39}$ A. Vaknin, Z. Ovadyahu, and M. Pollak, Phys. Rev. Lett. 84, 3402 (2000); Phys. Rev. B 65, 134208 (2002). 